\title{
Evidence for Informed Preservation Planning and Advocacy: A Synoptic View
}

Nancy Bell, National Archives and Record Administration, Washington DC, USA

May Cassar, Institute for Sustainable Heritage, University College London, UK

Matija Strlič, Institute for Sustainable Heritage, University College London, UK

\begin{abstract}
We argue the need to demonstrate the value of preservation services to institutions, the public and funders, because it matters conceptually and needs to be supported and validated. To address this imperative, improved and focussed data capture and its use by collection managers is advocated as the necessary first step towards the development of an evidence-based culture for preservation services and synoptic decision making, where evidence enables us to optimise the outcome of planning.
\end{abstract}

To do so, we need tools that enable us to evaluate diverse preservation scenarios. We demonstrate the potential of emerging cross-disciplinary tools and protocols developed in the recent years such as attitude surveys, computational modelling and demographic modelling. These offer evidence that informs prospective preservation planning, and, importantly, provides a credible evidence base for advocacy. These possibilities are discussed within the wider context of well-established preservation planning protocols that have shaped collection management for three decades, broadly characterised as retrospective.

\section{Introduction}

Preservation planning could predominantly be described as incremental (Mitchell, 2002). When faced with complexities, this type of decision making makes use of resources such that the solution is practically attainable and generally satisfactory to those impacted by the decision. It is likely that in such contexts not all scenarios can be considered as the complexities are considered to be too high. The incremental approach has a number of further features, such as:

1. There is no single correct solution as the end goal is often not well defined (e.g. the longterm horizon in preservation is often defined as "forever" or "as long as possible")

2. The problem is frequently redefined and end goals are adjusted to fit the available means

3. Decisions are designed to avoid negative impacts rather than to achieve desirable ones

4. The decision (and policy) process is ongoing and never ends.

There are positive and less positive aspects of incremental decision making. It could be seen as realistic and pragmatic, as it rarely moves away from accepted practice, however, in doing so, it rarely considers radical shifts in policy if conditions change markedly. Do the precarious economic and social situation in many countries and the impending climate change require of us to consider more radical approaches to decision making?

Accepting the principles of economic efficiency, synoptic planning attempts to optimise returns. It is based on the following steps: (i) Problem definition, (ii) Goal definition, (iii) Options appraisal, (iv) Choice of the optimal solution, (iv) Outcome monitoring. The most important criticism of synoptic 
planning is that in some contexts there is not enough data and certainty to follow the above steps and make the optimal decision.

It could be argued that incremental and synoptic decision making both have their strengths and weaknesses. It is probable that a mixed approach could best suit the complexities of preservation planning because incremental models will adapt to multiple goals, while synoptic models are better suited to achieving specific goals. However, in times of major societal and economic shifts synoptic planning is likely to perform better.

Over the last 30 years a number of tools, models and decision making frameworks have been developed to support 'rational decision making' for better collection management and preservation practice (Henderson and Waller, 2016). While risk assessment models, benchmarking practice, logic models, condition surveys (Taylor, 2014; Bell \& Lindsay, 2000; Cassar, 1998) have usefully informed collection management, funding decisions and priority setting, the focus was largely retrospective in design, mainly assessing historic damage against established qualitative criteria. In the recent years, attempts to reduce subjectivity and incorporate scientific validity through better data capture have gained ground (Duran et al., 2017). While these established methods have guided practice and informed policy for several decades, difficulties in aggregating data and restricted scalability hinders wider application of this important data to, for example, other organisations or to demonstrate the value economically, socially, and environmentally of preservation services to organisations and funders.

Why is this important? Increasingly, publicly and privately funded collecting institutions find themselves situated in a prevailing 'audit culture' of 'institutionalised expectations and instruments used to define benefits and returns on investment and to set priorities gained ground in the 1990s. This culture, described as a 'specific epoch' in Western international affairs with 'twinned precepts' of economic efficiency and good practice, has become a way of operating in most publically funded institutions, is not without opponents (Strathern, 2000,3). Critics view this culture of validation of ever-increasing accountability as another means of eclipsing intellectual endeavour and creativity, and therefore is to be avoided. Regardless of one's position on this divide, the epoch prevails in much of the Western world and preservation services are not immune from this culture. In the face of increased competition for resources and the demands of conflicting stakeholder priorities preservation services are sometimes viewed as a 'background' or a 'service' activity with a narrow remit are vulnerable in the face of conflicting 'front-line' services. To ensure a sustainable future, we need to get better at demonstrating the value of these services in ways that have meaning for stakeholders: decision makers, the public, the funders. Demonstrating the cultural, economic, social, and environmental value of these services offers a compelling argument to stakeholders, as these are the four pillars of sustainability that effectively capture an ecosystem that has application in the preservation domain. ${ }^{i}$

It is against this background that we argue for a paradigm shift that positions the use of evidence as central to synoptic decision making in preservation planning, and to demonstrating value by exploiting trans-disciplinary tools and methods.

\section{Problem definition}

Institutional policies and professional expertise mostly define what is inherently worthy of preservation and implicitly includes associated values of what is important to preserve and why (Eastop et al., 2012). However, in an audit culture, the public is an important stakeholder. Few studies have explored the attitudes of heritage users quantitatively, in the way that the outcomes of 
such studies could be used in modelling. In the 'Collections Demography' project ${ }^{\mathrm{ii}}$ tools have been developed that enabled the understanding of what public users value in the process of interaction with heritage. These tools are based on social science research methods and on attitude questionnaires, and provide us with a unique understanding of the values that users project on heritage, whether material or digital.

In the project, extensive evaluation of archive and library collections was carried out using an attitude questionnaire surveying 543 users (Dillon et al., 2012). The aim was to understand the value and meaning that are ascribed to documents. The values that emerged to be of importance, were (i) Future value, (ii) Materials \& sensory experience, (iii) Public value \& evidence, (iv) Personal meaning \& identity, (v) Understanding the present, (vi) Discovery \& engagement, (vii) Content \& learning, (viii) Connection to the past, (ix) Rarity. The vast majority of these can be preserved by preserving textual information, except (ii), which requires preservation of the material evidence itself, and rarity, which gives pleasure through visual, tactile and olfactory interactions with archive and library collections.

More recently, research at the Natural History Museum, London (Robb et al., 2013), explored expert stakeholders' values associated with geological collections. The stakeholder included 201 curators, academic researchers, subject specialist volunteers, conservators, archivists, and non-professional mineralogists. Six values were identified using an attitude questionnaire and exploratory factor analysis: (i) Personal/inspirational, (ii) Uniqueness, (iii) Originality/historic, (iv) Educational/future, (v) Aesthetic/Commercial, (vi) Information. The study concluded that the values (except Aesthetic/Commercial) relied heavily on 'contextual information' associated with a specimen (i.e. metadata), but not directly contained within the specimen (such as where the specimen was collected, by whom and when, storage notes and labels). When assessing the loss in value, the study indicated that loss of contextual information may be more detrimental than physical degradation of the specimen. This questions whether conservation of metadata archives is receiving appropriate conservation attention.

The two case studies demonstrate how attitude questionnaires can provide evidence that helps to define the preservation goal, and guide organisational decision making engaging both public users of heritage and experts. At the same time, these questionnaires can be used as engagement tools, and increase public understanding of the challenges associated with preservation. Eliciting value judgements from the public provides credible data to support greater transparency in decision making, thus increasing trust in the publically funded institutions creating public value, or value created by institutions (Bennington \& Moore, 2011).

\section{Goal definition}

In keeping with the principles of sustainability, intergenerational equity requires that we exploit heritage resources in a way that the benefits extracted through use (which can lead to resource loss, e.g. by increased handling or display) do not exhaust the resource and reduce future access.

From the perspective of normative planning (i.e. what should be done) it is currently unclear how as a community we go about defining planning horizons. Risk management typically operates with $100-$ 300-year horizons, and in Ashley-Smith's work on light damage, 50 years is used as a planning horizon within which just noticeable colour difference is considered to be acceptable.

In the work discussed in the previous section, library and archival users (Dillon et al., 2012) and mineralogical collection experts (Robb et al., 2013) were consulted on how long they would appreciate having collection items available in a state that is suitable for use (e.g. reading, handling, 
scientific investigation). The responses (Fig. 1) thus reflect both members of the engaged public (i.e. users of heritage) and experts, and reflect attitudes to both generally unstable organic and generally stable mineralogical collections. Interestingly, the response patterns are essentially the same, with cumulatively $\sim 50 \%$ of respondents being satisfied with a 100 -year planning horizon, and $\sim 80 \%$ with a 500 -year planning horizon.

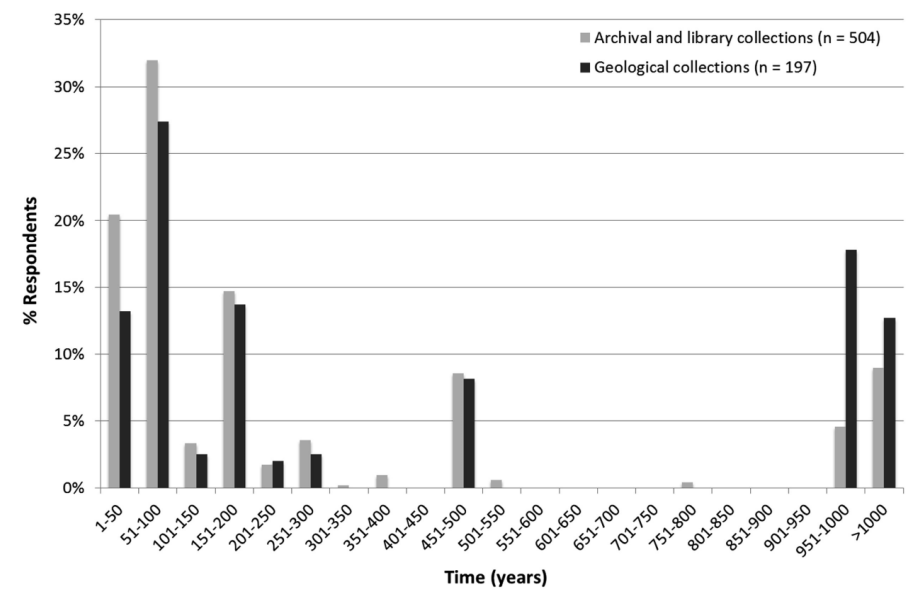

Fig. 1: Proportion of respondents (public users of archival and library collections, and expert users of mineralogical collections) as a function of acceptable long-term planning horizon expressed as time until loss of fitness for use.

However, different stakeholders operate in different temporalities, operating simultaneously in historic, current, and future time. From the above examples, it appears that the public and preservation professionals and practitioners operate in a temporal space of $\sim 100-500$ years, reflecting low discounting rates (Michalski, 2008) not unlike those typical for large infrastructure investments (Johnson, 2017) or environmental decision-making (Stern, 2006).

These long planning horizons may not be easily applicable at the levels of strategic planning (i.e. what could be done) and operational planning (i.e. what can be done) (Mitchel, 2002). Operational planning and allocation of financial resources is typically done in periods of time during which offices are held, e.g. a chief executive: 3-5 years, a government: 4-5 years. In strategic resource planning, e.g. in real estate (U.S. Code: Title 26 - Internal Revenue Code), depreciation periods range from 30 to 40 years for residential and non-residential properties, respectively. These overlapping and sometime conflicting temporalities (reflecting differet drivers involved in planning) need to be understood and reflected when setting preservation priorities and negotiated in view of economic and political imperatives, and public sensitivities, however, scenarios need to reflect normative, strategic and operational plans in order to provide evidence for all relevant contexts of decision making and stakeholders.

Table 1: Proposed planning horizons for different contexts of preventive conservation decision making.

\begin{tabular}{|l|l|}
\hline Type of planning & Period \\
\hline Normative (Planning for inter-generational equity) & 100 years \\
\hline Strategic (Planning large estates/infrastructure projects) & 30 years \\
\hline Operational (Short-term project planning) & 5 years \\
\hline
\end{tabular}


The temporal boundary represents one goal that requires definition; we now need to define what constitutes loss of fitness for use that might be unacceptable. This may or may not reflect the 'lifetime' of an object or of a collection, as loss of fitness might simply indicate that a conservation intervention or another resource-intensive action (e.g. handling under active supervision, or visual access using imaging technologies) is needed in order to ensure continued access to the heritage resource.

In the context of library and archival use, a public experiment involving 331 readers and visitors defined the threshold loss of fitness as the state of an object when text becomes missing (Strlič, 2015a). Similar psychophysical experiments were used previously for the assessment of the fitness for use of colour photographs (Ashley-Smith et al., 2002; Fenech et al., 2013).

\section{Options appraisal}

Both observational and experimental studies may lead to quantitative evidence that could enable us to develop options and assess them against the goals as defined in the previous section.

Observational (epidemiological) studies use collection survey methods and statistical methods of data analysis in order to derive observational data that can reveal risk factors leading to degradation, and possibly rates of change. There is a growing interest in observational data that could validate experimental results (Van Duin, 2014); however, such data is often highly noisy due to uncertainties related to sampling and measurement as well as unknown object histories. In a recent reliability engineering-based survey at the Amsterdam City Archives (Duran et al., 2017) factors that lead to accumulation of damage during access to archival materials have been quantified. In contrast to the usual collection surveys that aim to identify proportions of damaged material to assess conservation needs, reliability engineering-based surveys aim to identify causal relationships between exposure variables (design, maintenance, usage and manufacture) and outcomes (failure).

Experimental studies typically aim to develop mathematical models describing the quantitative relationship between change (related to fitness for use), material and environmental variables, and time (dose-response functions). Despite numerous studies, only a few such models have been developed that extend beyond single items and take into account structural and compositional variability across a collection, which is necessary in order to model the behaviour of an entire collection. Examples include colour photographs (Fenech et al., 2013), historic paper (Strlič et al., 2015a, 2015b, 2015c), and painting canvases (Oriola et al., 2015).

In the methodology that has become known as 'collections demography', collection surveys are performed with the specific goal to collect data that is required to model future degradation of a collection using dose response or damage functions. Demographic models (Fig. 2) essentially represent one half of the well-known population pyramid - a function of fitness for use over time. Depending on the dose-response function parameters, such demographic plots enable us to explore preservation options and assess preservation outcomes.

Figure 2 shows selected demographic curves describing the degradation of painting canvases (i.e. fabric) as a function of canvas acidity, environmental temperature and humidity, and time. In comparison with the status quo (storage at average conditions of $21^{\circ} \mathrm{C}, 58 \% \mathrm{RH}$ ), storage at a lower temperature $\left(15^{\circ} \mathrm{C}, 50 \% \mathrm{RH}\right)$ would considerably increase the fitness of canvases for travel, e.g. to exhibitions. Such environmental conditions could be achieved by keeping the collection in passive 
storage (Ryhl-Svendsen et al., 2012), which would additionally reduce carbon emission. If such conditions cannot be ensured, then deacidification could be explored as a conservation intervention.

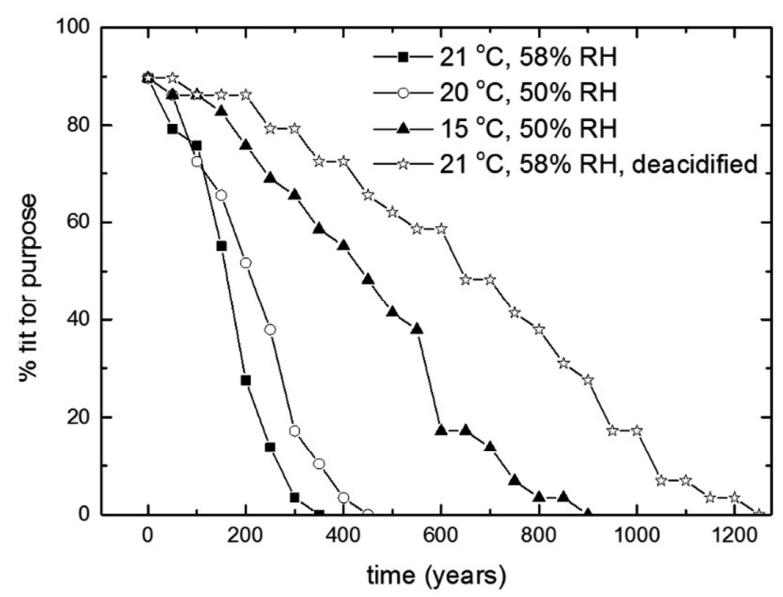

Fig. 2: Demographic plots for the surveyed collection of paintings at the National Museum of Art of Catalonia, Barcelona, at the actual environmental conditions $\left(21^{\circ} \mathrm{C}, 58 \% \mathrm{RH}\right)$ as well as three hypothetic preservation scenarios (Oriola et al., 2015). For the purpose of this research fitness for purpose was defined as threshold physical strength of canvas that can withstand transportation without a significant risk of mechanical damage.

The options appraisal as depicted in Fig. 2 is associated with more than just investment in terms of financial value. It could be argued that deacidification is the least costly solution in monetary terms, as it requires neither a capital investment nor a continuous investment into climate control, and can be discounted over the entire lifetime of an object (i.e. until the next conservation intervention such as lining or another method of canvas reinforcement). However, deacidification changes the chemical composition of the canvas (and thus its information value), and conservation ethics aspects plays an important role in such options appraisals.

\section{Choice of the optimal solution}

As an example of synoptic decision making in preservation where the optimal choice has been carefully and openly justified, the Building Environment Simulation (BES) project was a collaborative 22-month study between The National Archives (UK) and UCL Institute for Sustainable Heritage. It offers an illustration of the value of computational modelling to generate scenarios that can transform preservation policy and deliver impact beyond the organisation.

This research was highly effective as in the process of problem definition and definition of goals, discussions involved stakeholders within the institution as diverse as conservators, collection managers, scientists and facility managers. High energy use and carbon emissions associated with collection storage environment were defined as the problem (Directive 2010/31/EU) and the optimal solution would define operating conditions that would improve the overall preservation outcome while at the same time reduce the energy cost and mitigate any predicted consequences of climate change.

Collaboration was established with building scientists using the computational modelling tool Energy+ to understand the environmental behaviour of the building and of the climate control 
system. Environmental records collected over many years, as well as the detail of the repository construction were used to build a model of three TNA repositories, based on their physical dimensions, material characteristics, mechanical air conditioning and relative humidity (RH) setting (Hong et al., 2012). The model was developed to an agreed accuracy level, against which various scenarios were tested in response to external environmental conditions, potential changes in the structure of the buildings, operation of the HVAC system, quantity (mass) of the content of the repositories, and other changes relating to the occupancy and the use of the building.

Table 2: Environmental management scenario appraisal in the BES project (Hong et al., 2012). 'Baseline' represents the environmental scenario as was applied in 2009, prior to any changes. 'Weekend power down' scenario assumed running the HVAC during weekdays only, while 'Seasonal adjustment' assumed incremental changes in the setpoints from $16^{\circ} \mathrm{C} / 35 \% \mathrm{RH}$ in February to 20 ${ }^{\circ} \mathrm{C} / 55 \% \mathrm{RH}$ in September and then back to $16^{\circ} \mathrm{C} / 35 \% \mathrm{RH}$ by December. The 'External climate' scenarios assumed climate to change in accordance with (UKCP09 UK Climate Projections: UKCP09), UK Climate Impacts Programme projections and the environmental set points to remain the same as per the 'Baseline' scenario.

\begin{tabular}{|l|c|c|}
\hline Scenario & Energy load (kWh/m2/year) & \% change relative to the Baseline \\
\hline Baseline & 33 & 0 \\
\hline Weekend power down & 29 & -12 \\
\hline Seasonal adjustment & 19 & -42 \\
\hline External climate in 2050 & 38 & +15 \\
\hline External climate in 2080 & 42 & +27 \\
\hline
\end{tabular}

Scenario appraisal clearly indicated that seasonal adjustment led to the most significant reduction in energy consumption while it achieved an increased collection lifetime at the same time. The saving was even predicted to offset the changes in energy use due to climate change as far into the future as 2080 .

\section{Monitoring the outcome}

Monitoring of planning outcomes is required in order to assess if goals have been achieved and whether adjustments to the selected options are needed. As discussed previously (Strlič et al., 2013) monitoring of outcomes is relatively straightforward if the goal is to achieve desired environmental conditions (e.g. in prescriptive standards), however, if the goal is to achieve desired object fitness (e.g. in performance standards), then monitoring of outcomes becomes associated with object surveys and thus significantly more resource intensive than environmental monitoring. Few standard monitoring procedures exist for this purpose (BS ISO 15928-3. 2009).

In addition to improved preservation, the BES project discussed in the previous section set itself the goal to reduce energy consumption and cost which was achieved, as was the goal to meet Government energy reduction targets: a 27.3\% reduction in CO2 emissions against the 2009/10 baseline was achieved in 2011/12, a monetary saving has been achieved by reduction in energy use by $53 \%$, and as a consequence, the Display Energy Certificate has improved (Fig. 3 ). 


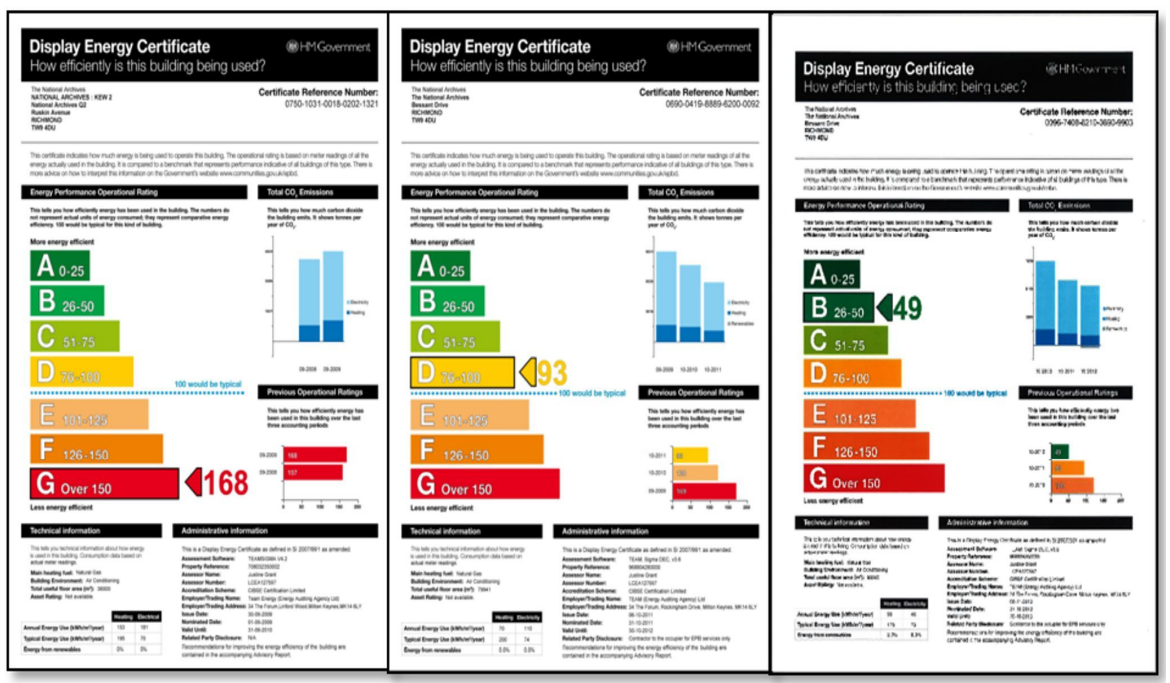

Fig. 3: The Display Energy Certificates for three consecutive years of 2009/10, 2010/11, 2011/2012 from left to right, as the outcome of the implementation of the BES project at the National Archives (UK).

\section{Conclusion}

Currently, incremental planning pervades preventive conservation. We thus explored the potential benefits of synoptic planning and explored the steps that define it. Existing notions of the significance and value of collections are challenged if users are seen as a stakeholder in the planning process, as would be expected in a democratic audit culture. Their expectations for current and future fitness for use added important layers to the long-established precepts underpinning preservation planning at The National Archives (UK) and as a result, information services were recalibrated and resources were directed to strategies to manage material change of collection items with predicted period till loss of fitness of 50 years or less.

Such re-alignment proved contentious as some conservation professionals questioned the influence of public perceptions of value and significance on preservation policy. However, armed with the evidence provided by the demographic model, the logic underpinning decisions was irrefutable. This same evidence gained favour within the organisation as it proved to be a valuable tool to demonstrate an efficient return on investment and therefore could demonstrate economic benefits.

As a particular example of a project that adopted synoptic planning from start to execution, the Building Environment Simulation project at the National Archives (UK) was singled out. The project offers a compelling illustration of the value of computational modelling to generate data to transform preservation policy and deliver impact, or change, beyond the organisation (Ntanos \& Bell, 2007). The findings in this project established that a thorough understanding of building performances based on evidence challenged existing organisational myths, which led to a refreshed capital programme for environmental upgrades. As a consequence, the economic value of research and improved environmental standards demonstrated environmental value.

The findings informed a change in environmental management of collections, and gave rise to a new environmental standard PAS:198: Environmental Guidelines for Cultural Heritage Collections (PAS:198 2012) that incorporates a risk-based approach to managing the collection environment with reference to the needs of the collection, intended use, expected lifetime and energy economy. 
Exploitation of cross-disciplinary methods is essential for building quantitative and qualitative evidence for future preservation planning and this should be the basis for developing an evidence based culture for preservation services in the coming decades. This position sits within the overarching theme that emerges from this multifaceted and multidisciplinary narrative, and reinforces the view that preservation activities add social, cultural, economic and environmental values that support the pillars of sustainability and can be used as an important tool for advocating the social and economic value of preservation programmes.

\section{References}

Ashley-Smith, J., A. Derbyshire, B. Pretzel. 2002. "The continuing development of a practical lighting policy for works of art on paper and other object types at the Victoria and Albert Museum". Postprints of the ICOM-CC $13^{\text {th }}$ Triennial Meeting, Rio de Janeiro. London: James \& James, pp.3-8.

Bell, N., H. Lindsay. 2000. "Benchmarks in Collection Care for UK Libraries”. Library Information Services, Yorkshire.

Benington, J. and M. H. Moore (Eds.). 2011. Public value: theory and practice. Basingstoke: Palgrave Macmillan.

British Standards Institute (2012), Publically Available Specification: 198 Specification for managing environmental conditions for cultural collections. London: British Standards Institution

BS ISO 15928-3. 2009. Houses - Description of Performance - Part 3: Structural Durability. London: British Standards Institute.

Cassar, M. 1998. Cost/Benefit Appraisals for Collections: A Practical Guide, London: Museums and Galleries, pp. $1-48$.

Dillon, C., W. Lindsay, J. Taylor, K. Fouseki, N. Bell, M. Strlič. 2013. “Collections demography: stakeholders' views on the lifetime of collections". Postprints of the Munich Climate Conference 7 to 9 November 2012, Climate for Collections, Standards and Uncertainties, pp. 45-58.

Directive 2010/31/EU of the European Parliament and of the Council of 19 May 2010 on the energy performance of buildings. Official Journal of the European Union, 2010.

Duran, C., J. Grau, T. Fearn, M. Strlič. 2017. "Data mining in collections: from epidemiology to demography". Preprints of the 18th International Conference of the International Council of Museums Committee for Conservation, Copenhagen, Denmark, 4-8 Sep 2017.

Eastop, D., A. E. Bülow, A. W. Brokerhof. 2012. "Design, digitization, discovery: Enhancing collection quality", Studies in Conservation, 57:sup1, S102-S96.

Fenech, A., C. Dillon, K. Ntanos, N. Bell, M. Barrett, M. Strlič. 2013. "Modelling the Lifetime of Colour Photographs in Archival Collections", Stud. Conserv., 58, 107-116.

Henderson, J., R. Waller. 2016. "Effective preservation decision strategies", Stud. Conserv., 61, 308-323.

Hong, S. H., M. Strlič, I. Ridley, K. Ntanos, N., Bell, M. Cassar. 2011. “Monitoring and modelling the storage environment at The National Archives, UK". Preprints of the $16^{\text {th }}$ Triennial Conference Lisbon, 19-23 September 2011.

Hong, S. H., M. Strlič, I., Ridley, K. Ntanos, N. Bell, M. Cassar. 2012. "Climate change mitigation strategies for mechanically controlled repositories: the case of The National Archives, Kew". Atm. Env., 49, 163-170.

Johnsen, J. S., M. Cassar, D. Saunders. 2017. "Risk assessment for the relocation of the historical Viking ships from Bygdøy, Oslo, Norway". Preprints of the $18^{\text {th }}$ Triennial Conference, Copenhagen, 4-8 September 2017.

Michalski, S. 2008). "Social discount rate: modelling collection value to future generations, and understanding the difference between short-term and long-term preservation actions". Preprints of the $15^{\text {th }}$ Triennial Conference, New Delhi, 22-26 September 2008, pp. 751-758.

Mitchell, B. 2002. Resource and Environmental Management. Prentice Hall, Harlow. 
Ntanos, K., N. Bell. 2007. "A holistic appraisal of environmental conditions in The National Archives, UK". Museum microclimates: contributions to the Copenhagen conference, Copenhagen, Denmark, 19-23 November 2007.

Oriola, M., G. Campo, C. Ruiz-Recasens, N. Pedragosa, M. Strlič. 2015. “Conservation management scenario appraisal for painting canvases at Museu Nacional d'Art de Catalunya", Stud. Conserv., 60, S193-S199.

Robb, J., C. Dillon, M. Rumsey, M. Strlič. 2013. “Quantitative Assessment of Perceived Value of Geological Collections by 'Experts' for Improved Collections Management". The Geological Curator, 9 (10), 529-543.

Ryhl-Svendsen, M., L. Aasbjerg Jensen, P. Klenz Larsen, B. Bøhm, T. Padfield. 2013. "A museum storage facility controlled by solar energy". Postprints of the Munich Climate Conference 7 to 9 November 2012, Climate for Collections, Standards and Uncertainties, pp. 141-149.

Stern, N. 2006. Stern Review on the Economics of Climate Change. London: HM Treasury.

Strathern, M. (2000): “Introduction: new accountabilities". In: M. Strathern (Ed.) Audit Cultures. Anthropological studies in accountability, ethics and the academy, Routledge, London.

Strlič, M., D. Thickett, J. Taylor, M. Cassar. 2013. "Damage functions in heritage science”, Stud. Conserv., 58, 80-87.

Strlič, M., C. Grossi-Sampedro, C. Dillon, N. Bell, K. Fouseki, P. Brimblecombe, E. Menart, K. Ntanos, W. Lindsay, D. Thickett, F. France, G. De Bruin. 2015a. "Damage Function for Historic Paper. Part I: Fitness for Use", Her. Sci. $3, \# 33$.

Strlič, M., C. Grossi-Sampedro, C. Dillon, N. Bell, K. Fouseki, P. Brimblecombe, E. Menart, K. Ntanos, W. Lindsay, D. Thickett, F. France, G. De Bruin. 2015b. "Damage Function for Historic Paper. Part II: Wear and Tear", Her. Sci. 3 , \#36.

Strlič, M., C. Grossi-Sampedro, C. Dillon, N. Bell, K. Fouseki, P. Brimblecombe, E. Menart, K. Ntanos, W. Lindsay, D. Thickett, F. France, G. De Bruin, 2015c. "Damage Function for Historic Paper. Part III: Isochrones and Demography of Collections", Her. Sci. 3, \#40.

Taylor, J. 2013. "An integrated Approach to Risk Assessment and Condition Surveys". Journal of the American Institute for Conservation, 127-141.

UK Climate Projections (UKCP09), UK Climate Impacts Programme, http://www.ukcip.org.uk/index.php

U.S. Code: Title 26 - Internal Revenue Code, $§ 168$ - Accelerated cost recovery system, https://www.law.cornell.edu/uscode/text/26/168

Van Duin P. 2014. "Climate Effects on museum objects: The need for monitoring and analysis". GCI Newsletter 29, 13-15.

Waller, R. 1994. "Conservation risk assessment: A strategy for managing resources for preventive conservation". Preprints of the Contributions to the Ottawa Congress, 1216 September 1994, Preventive Conservation: Practice, Theory and Research. IIC, London, pp. 12-16.

'The concept of 'pillars of sustainability' offers useful theoretical frame for the preservation domain. See for example: http://www.circularecology.com/sustainability-and-sustainable-development.html\#.Wdo 7Xn2a1s ii For a full description of the project see: https://www.ucl.ac.uk/bartlett/heritage/research/projects/projectarchive/collections-demography-dynamic-evolution-populations-objects 